\title{
Voluntary attention increases perceived spatial frequency
}

\author{
JARed Abrams, Antoine Barbot, and Marisa Carrasco \\ New York University, New York, New York
}

\begin{abstract}
Voluntary covert attention selects relevant sensory information for prioritized processing. The behavioral and neural consequences of such selection have been extensively documented, but its phenomenology has received little empirical investigation. Involuntary attention increases perceived spatial frequency (Gobell \& Carrasco, 2005), but involuntary attention can differ from voluntary attention in its effects on performance in tasks mediated by spatial resolution (Yeshurun, Montagna, \& Carrasco, 2008). Therefore, we ask whether voluntary attention affects the subjective appearance of spatial frequency-a fundamental dimension of visual perception underlying spatial resolution. We used a demanding rapid serial visual presentation task to direct voluntary attention and measured perceived spatial frequency at the attended and unattended locations. Attention increased the perceived spatial frequency of suprathreshold stimuli and also improved performance on a concurrent orientation discrimination task. In the control experiment, we ruled out response bias as an alternative account by using a lengthened interstimulus interval, which allows observers to disengage attention from the cued location. In contrast to the main experiment, the observers showed neither increased perceived spatial frequency nor improved orientation discrimination at the attended location. Thus, this study establishes that voluntary attention increases perceived spatial frequency. This phenomenological consequence links behavioral and neurophysiological studies on the effects of attention.
\end{abstract}

Whenever we open our eyes, we face an overwhelming amount of information. Despite this fact, we seem to have a clear understanding of what we see. Attention helps us overcome this challenge by selecting relevant information for prioritized processing. When spatial attention is deployed to a specific location in a visual scene, objects appearing at relevant locations receive enhanced representation, whereas objects appearing at irrelevant locations are underrepresented. Some authors have related the benefits and costs of attention to the high cost of cortical computation (e.g., Pestilli \& Carrasco, 2005). Because of metabolic limitations within the brain, only a small population of neurons can become significantly more active at a given time (Lennie, 2003). Attention optimizes the brain's use of its limited resources by prioritizing some aspects of the visual scene and reducing the processing of others. Recent studies have shown that these benefits and costs are reflected behaviorally in terms of both performance and appearance (e.g., Carrasco, Ling, \& Read, 2004; Giordano, McElree, \& Carrasco, 2009; Ling \& Carrasco, 2006b; Liu, Abrams, \& Carrasco, 2009; Montagna, Pestilli, \& Carrasco, 2009; Pestilli \& Carrasco, 2005; Pestilli, Viera, \& Carrasco, 2007).

\section{Visual Attention}

Visual attention can be deployed either reflexively, in response to sudden stimulation, or voluntarily, in response to goals or task demands-exogenously or endogenously, respectively. Involuntary (exogenous) attention peaks in about $100 \mathrm{msec}$, decays rapidly thereafter, and is generally engaged by brief, peripheral cues. Voluntary (endogenous) attention takes approximately $300 \mathrm{msec}$ to be deployed, can be sustained for several seconds, and is engaged by symbolic cues (Müller \& Rabbitt, 1989; Nakayama \& Mackeben, 1989; Posner, 1980; Yantis \& Jonides, 1990).

Both voluntary and involuntary attention enhance contrast sensitivity, spatial resolution, and acuity at cued locations, while carrying a cost at uncued locations, with respect to a neutral baseline (Montagna et al., 2009; Pestilli \& Carrasco, 2005; Pestilli et al., 2007; for reviews, see Carrasco, 2006, 2009a; Carrasco \& Yeshurun, 2009). Correspondingly, both types also speed information accrual at cued locations while slowing accrual at uncued locations (Carrasco \& McElree, 2001; Carrasco, Giordano, \& McElree, 2004, 2006; Giordano et al., 2009). Attention allows observers to perform more difficult discriminations, but this enhancement leads to a detriment in performance at unattended locations. Furthermore, both types of attention increase perceived contrast (Carrasco, Fuller, \& Ling, 2008; Carrasco, Ling, \& Read, 2004; Fuller, Park, \& Carrasco, 2009; Ling \& Carrasco, 2007; Liu et al., 2009). That is, attention not only makes stimuli easier to discriminate, but also affects the subjective experience, making those stimuli look higher in contrast. However, these types of attention can have different behavioral effects. For instance, both types of attention enhance performance in tasks de-

M. Carrasco, marisa.carrasco@nyu.edu 
pendent on contrast sensitivity (Ling \& Carrasco, 2006a; Lu \& Dosher, 2000; Pestilli, Ling, \& Carrasco, 2009) and texture segmentation (Yeshurun, Montagna, \& Carrasco, 2008), but the two types of attention seem to do so via different mechanisms.

Involuntary attention has been shown to increase perceived spatial frequency and gap size (Gobell \& Carrasco, 2005), but the effects of voluntary attention on perceived spatial frequency have not been characterized. Whereas some researchers have shown that voluntary spatial attention increases perceived contrast (Liu et al., 2009) and brightness (Tse, 2005), others have reported that increasing the availability of attentional resources does not alter appearance of these dimensions, but only reduces response variability (Prinzmetal, Amiri, Allen, \& Edwards, 1998; Prinzmetal, Nwachuku, Bodanski, Blumenfeld, \& Shimizu, 1997). Thus, it is necessary to empirically assess the effects of voluntary attention on perceived spatial frequency to have a fuller understanding of the way covert attention may alter appearance.

\section{Apparent Spatial Frequency}

Using the $2 \times 2$ alternative forced choice (AFC) task developed to assess the effects of attention on contrast appearance (Carrasco, Ling, \& Read, 2004), Gobell and Carrasco (2005) found that involuntary attention increases both apparent spatial frequency and gap size. This paradigm makes it possible to study subjective experience objectively and rigorously (Luck, 2004; Treue, 2004). Gobell and Carrasco argued that these effects of involuntary attention were consistent with its role in increasing spatial resolution. We foresee two hypotheses regarding the effects of voluntary attention on perceived frequency.

On one hand, voluntary attention could operate in a fashion similar to that of involuntary attention, enhancing the apparent spatial frequency of an attended stimulus (Gobell \& Carrasco, 2005). Involuntary attention always increases spatial resolution in a texture segmentation task; it improves performance where resolution is low, but it impairs performance where resolution is already too high for the task at hand (Talgar \& Carrasco, 2002; Yeshurun \& Carrasco, 1998, 2000, 2008; Yeshurun et al., 2008). Correspondingly, when observers are selectively adapted to high but not to low spatial frequencies, the cost of involuntary attention is abolished. This finding suggests that involuntary attention reweights the spatial frequency population response, shifting sensitivity toward higher spatial frequency content, and does so in an inflexible manner (Carrasco, Loula, \& Ho, 2006).

On the other hand, given the flexible nature of voluntary attention, it may not alter apparent spatial frequency. For instance, voluntary attention improves performance in a texture segmentation task, regardless of the resolution of the task at hand (Yeshurun et al., 2008), and whereas the deployment of involuntary attention is automatic and inflexible, when observers deploy voluntary attention they are able to do so as a function of cue validity (Giordano et al., 2009). Furthermore, an endogenous mechanism in macaque MT in the form of shifting and shrinking receptive fields around the attended stimulus has been reported in neurophysiological studies (Anton-Erxleben, Stephan, \& Treue, 2009; Womelsdorf, Anton-Erxleben, Pieper, \& Treue, 2006). Such a mechanism could result in an enhanced representation without necessarily shifting the average global population response toward higher spatial frequencies. Consequently, it is plausible that voluntary attention would optimize performance while leaving appearance unchanged. Such a dissociation has been shown with the effects of involuntary attention on hue (Fuller \& Carrasco, 2006).

To assess the effects of voluntary attention on apparent spatial frequency, we used a procedure similar to Gobell and Carrasco's (2005) with involuntary attention. To manipulate voluntary attention, we utilized the rapid serial visual presentation (RSVP) paradigm, which was used in a recent study to characterize the effect of voluntary attention on contrast sensitivity (Liu et al., 2009).

\section{METHOD}

\section{Main Experiment}

In order to direct observers' voluntary attention, we adopted the RSVP method implemented by Liu et al. (2009), in which observers were asked to detect a target letter (X) presented among a stream of distractor letters. Two streams were presented, one on each side of the display, and the observers were cued either to a single stream or to both streams. The cue indicated the side of the space where the target letter would appear but not whether it would appear on a given trial. This method has the advantage of directing voluntary attention, via a central cue, in a way that is unrelated to the appearance task. Consequently, the cue has no bearing on the appearance judgment and is unlikely to induce a bias. Using the $2 \times 2 \mathrm{AFC}$ task combined with RSVP, in this study, we investigated the effects of voluntary attention on perceived spatial frequency. To confirm the role of attention and rule out a response bias, we lengthened the interval between the RSVP stream and the $2 \times 2$ AFC task, allowing the observers to disengage from the originally attended location and to return to a neutral attentional state. Previous research on attentional dwell time has demonstrated that it takes about $250 \mathrm{msec}$ to disengage voluntary attention (Horowitz, Wolfe, Alvarez, Cohen, \& Kuzmova, 2009; Theeuwes, Godijn, \& Pratt, 2004). Therefore, we predicted that if attention would alter perceived spatial frequency and enhance orientation discrimination, it would do so with the short but not with the long interval. Specifically, attention would still be present at the short interval, leading to an increase in perceived spatial frequency and better orientation discrimination, whereas it would no longer be at the cued location at the long interval, leading to a change in neither the perceived spatial frequency nor orientation discrimination performance. Moreover, if the cuing led to an increase in perceived spatial frequency with the long interval without a persisting increase in orientation discrimination performance, this would be clear evidence of a bias. Thus, the control experiment allows for the reliable assessment of both perceptual effects and bias effects.

Participants. Eight graduate students at New York University participated as observers in both experiments. Five were experienced psychophysical observers. All but two observers (authors) were naive as to the purpose of the experiment. All of the participants had normal or corrected-to-normal vision.

Apparatus. The stimuli were generated using MATLAB (MathWorks, Natick, MA) and MGL (http://justingardner.net/mgl) and were displayed on a 21-in. CRT monitor (with a resolution of $1,024 \times$ 768 pixels and a refresh rate of $100 \mathrm{~Hz}$ ). The display was calibrated using a Photo Research (Chatworth, CA) PR650 SpectraColorimeter to linearize the gamma. The observers' eye position was monitored using an infrared video camera system (ISCAN, Burlington, MA). Videos of the left eye were recorded and inspected offline to de- 


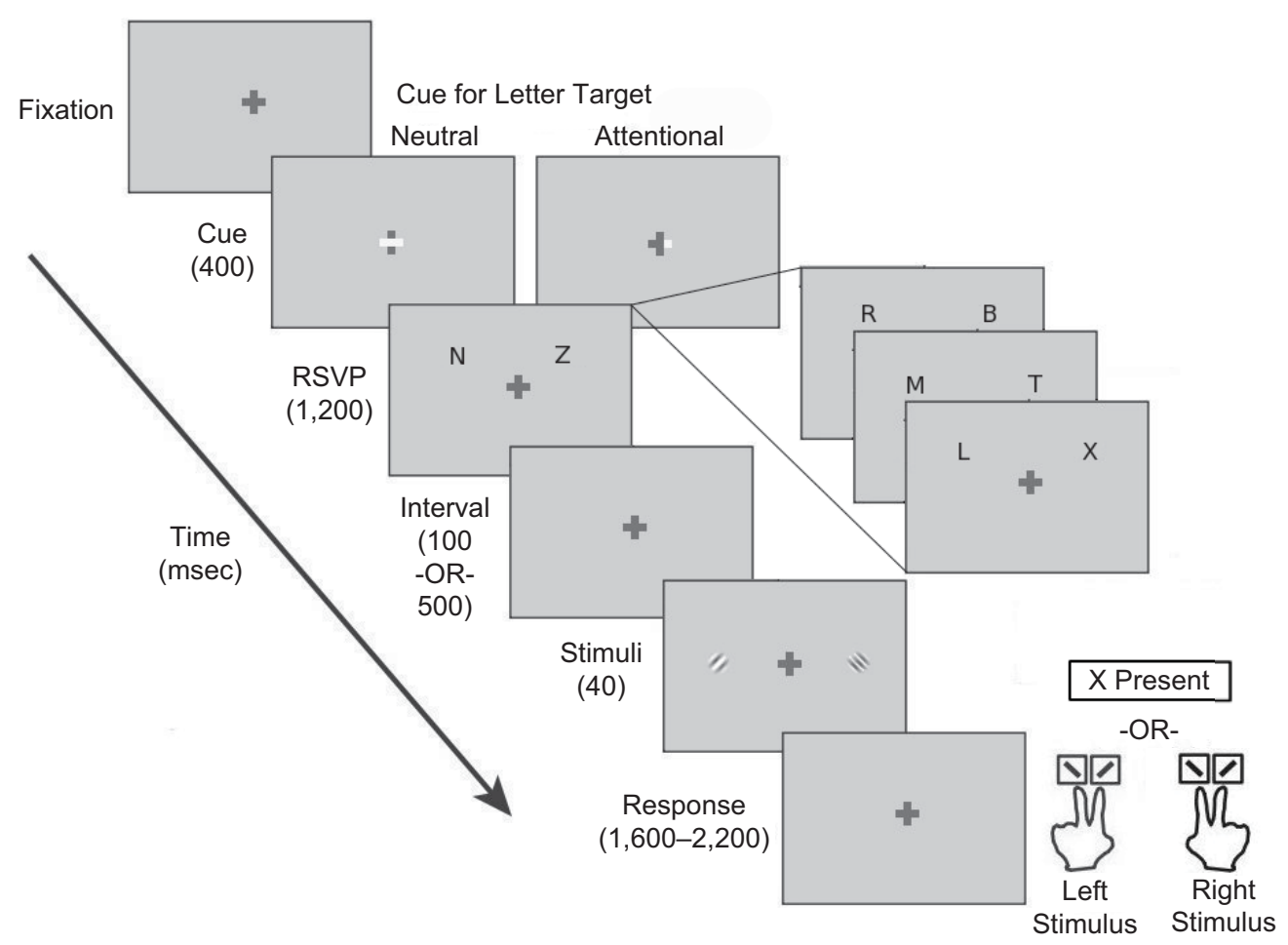

Figure 1. Schematic depiction of the trial sequence. For ease of illustration, the cues are depicted as a brightening of all or part of the horizontal bar of the fixation cross, whereas in the experiment, the cues were presented as a thickening of all or part of this bar. Also for illustrative purposes, the tilt of the Gabors is exaggerated, and only four rapid serial visual presentation (RSVP) frames are shown. The inset depicts the response scheme: If an X was present in the RSVP stream, the participants pressed the space bar; otherwise, they were asked to report the orientation of the Gabor stimulus with the higher spatial frequency, using the right hand if it was the Gabor on the right and the left hand if it was the Gabor on the left. For either hand, one of two possible keys was pressed to indicate whether the Gabor was tilted to the left (") $\mid$ ") or to the right ("“/”).

tect breaks from fixation. All of the observers were able to maintain steady fixation, breaking fixation in less than $1 \%$ of the trials.

Stimuli. There were four types of display in the experiment (Figure 1): fixation, cue, RSVP, and Gabor patches (sinusoidal gratings embedded in a Gaussian window). The background of the screen was gray $\left(50 \mathrm{~cd} / \mathrm{m}^{2}\right)$, and a centrally located cross $\left(0.5^{\circ} \times 0.5^{\circ}, 100 \mathrm{~cd} / \mathrm{m}^{2}\right)$ served as fixation. The cue was denoted by the thickening of one arm (attention cue) or both arms (neutral cue) of the horizontal bar of the fixation cross. The RSVP display consisted of two streams of letters (distractors: N, R, Z, B, A, M, L, T; target: X) located at $6^{\circ}$ eccentricity $\left(0.8^{\circ}\right.$ azimuth $)$. The offset from horizontal was to prevent forward masking of the Gabor patches by the letter streams (Yeshurun \& Carrasco, 1999). The letters were white $\left(100 \mathrm{~cd} / \mathrm{m}^{2}\right)$ and $0.6^{\circ}$ in size. The RSVP streams were synchronized, lasted for $1.2 \mathrm{sec}$, and contained between five and eight letters (adjusted blockwise for each observer so that performance remained at a $d^{\prime}$ of $\sim 1.5$ in the neutral cuing condition), with no blanks between the letters. In a given trial, the number of items per stream could be offset by \pm 1 to add temporal uncertainty and to optimize attentional deployment. On trials in which the target letter was present, it could occur in any frame in the stream, except for the first frame. The Gabor patches $\left(50 \%\right.$ contrast, subtended $2^{\circ}$ visual angle, $S D$ of the Gaussian window: $0.3^{\circ}$ ) were located on the horizontal meridian, centered at $6^{\circ}$ of eccentricity, with a small orientation offset from vertical $\left( \pm 1^{\circ}-5^{\circ}\right.$ adjusted for each observer so that neutral performance remained at $\sim 85 \%$ for discrimination accuracy). The phase of the Gabor patches was not varied, because the presentation time of the stimuli and the length of time between subsequent trials could not result in retinal adaptation. When observer RSVP performance was poor $\left(d^{\prime}<1\right)$, the observers ran additional blocks to replace them ( $<5 \%$ of total blocks). The observers viewed the display from a distance of $57 \mathrm{~cm}$ with their heads stabilized by a chinrest.

Procedure. A sample trial is shown in Figure 1. Following fixation, the cue appeared for $400 \mathrm{msec}$, followed by the presentation of the two RSVP streams for $1.2 \mathrm{sec}$. This was followed by a $100-\mathrm{msec}$ interstimulus interval (ISI), after which two Gabor patches appeared simultaneously for $40 \mathrm{msec}$. One of the Gabors was the standard ( $3.5 \mathrm{cpd})$, whereas the other was the test, presented at one of nine spatial frequencies $(2,2.5,3,3.25,3.5,3.75,4,4.5$, or $5 \mathrm{cpd})$ with equal probability.

We used an RSVP detection task to engage focal attention at one of the two locations. The observers were told to attend either to the cued RSVP stream (attention cue) or to both streams (neutral cue), to press the space bar if they detected the target letter X, and to not respond to the subsequent Gabor patches if the $\mathrm{X}$ was present. Half the trials were neutral cued, and the other half were attention cued with $100 \%$ cue validity, which referred to target location but not to its occurrence. On attention-cued trials, the target letter could only appear on the cued side. The difference in RSVP performance between the neutral-cued and attention-cued conditions is a confirmation of the effectiveness of the attentional manipulation; orientation discrimination and appearance are the variables of interest.

The target letter was present only on $20 \%$ of the trials (equally likely in the left and right locations). The observers were informed that the target was rare and told not to respond to target absence. Instead, when they did not see the target letter, they were instructed to report the orientation of the higher spatial frequency Gabor (i.e., the appearance judgment). The observers used one of four possible keys: the " $\mathrm{z}$ " and " $\mathrm{x}$ " keys for counterclockwise and clockwise 
tilted, respectively, if the left Gabor was of higher spatial frequency, and the " 1 " and " 2 " keys (on the numeric keypad of the keyboard) for counterclockwise and clockwise tilted, respectively, if the right Gabor was of higher spatial frequency. Thus, with a single key response, the observers indicated both the location and the orientation of the higher spatial frequency stimulus, giving a measure of both their appearance judgment and their discrimination performance. The observers were required to respond within the time allotted by a variable response window (1.6-2.2 sec). Cue location, Gabor orientation, and the locations of the test and standard stimuli were randomized on every trial. Furthermore, the observers were explicitly informed that the cue carried information only about the RSVP task, not about the orientation or spatial frequency judgment. The observers completed 1,080 trials in 12 blocks, with 6 blocks conducted per session (day).

The rationale for using the demanding RSVP task combined with a $100 \%$ valid attention cue is to encourage observers to attend to the cued location. The short ISI $(100 \mathrm{msec})$ between the RSVP and Gabors ensured that sustained attention was still directed to the peripheral location; previous research has demonstrated that attention takes about $250 \mathrm{msec}$ to disengage (Horowitz et al., 2009; Theeuwes et al., 2004).

\section{Control Experiment: Response Bias}

In this experiment, the procedure was identical to that of the main experiment, except that the ISI between the RSVP offset and the Gabor onset was long $(500 \mathrm{msec})$, rather than short $(100 \mathrm{msec})$. Whereas a 100-msec ISI is too short to disengage attention from the cued location, a 500-msec ISI provides ample time to redeploy attention (Horowitz et al., 2009; Posner, 1980; Theeuwes et al., 2004). Again, the observers completed 1,080 trials of the control experiment, divided into 12 blocks across two sessions (days). The order of the main and control experiments was counterbalanced across observers. None of the observers were informed that there were two different experiments across the four sessions. Since the observers were explicitly informed that the cue does not carry any information about the location or orientation of the stimulus of higher spatial frequency, the optimal strategy is to attempt to redeploy attention to both locations after the RSVP streams disappear. Thus, if the results in the main experiment were driven by response bias, the observers should choose the Gabor in the cued location more often, regardless of the ISI. Conversely, if the appearance or tilt discrimination effects were the result of attention, the effect should diminish or even disappear as the interval between the RSVP and the Gabor stimuli presentation is increased.

\section{RESULTS}

\section{RSVP Detection}

Main experiment and control experiment. We used the RSVP task to induce a focused state of attention to the cued location. Although performance on this task is not of main interest in our experiments, we present the data to show that attention was indeed effectively manipulated. Figures 2A and 2B show the average $d^{\prime}$ values in the neutral and attentional cue conditions, for the main (Figure 2A) and the control (Figure 2B) experiments. Detection performance was significantly better for the attentional cue than for the neutral cue conditions in both experiments [paired $t$ test, main experiment: $t(7)=9.48$, $p<.001, d=1.20$; control experiment: $t(7)=6.06, p<$ $.001, d=1.56]$. Thus, the RSVP task effectively manipulated the observers' attention, which was deployed to the peripheral location.

\section{Appearance}

Main experiment. Psychometric functions were fitted with a four-parameter Weibull function, $\psi=\gamma+(1-$ $\gamma-\lambda)\left\{1-\exp \left[-(x / \alpha)^{\beta}\right]\right\}$, where $\psi$ is the proportion of responses indicating that the test had higher spatial frequency than the standard; $x$ is the spatial frequency of the test stimulus; $\alpha$ is the location parameter; $\beta$ is the slope; and $\gamma$ and $\lambda$ are lower and upper asymptotes, respectively.

\section{A Main Experiment; $\mid \mathrm{SI}=100 \mathrm{msec}$}

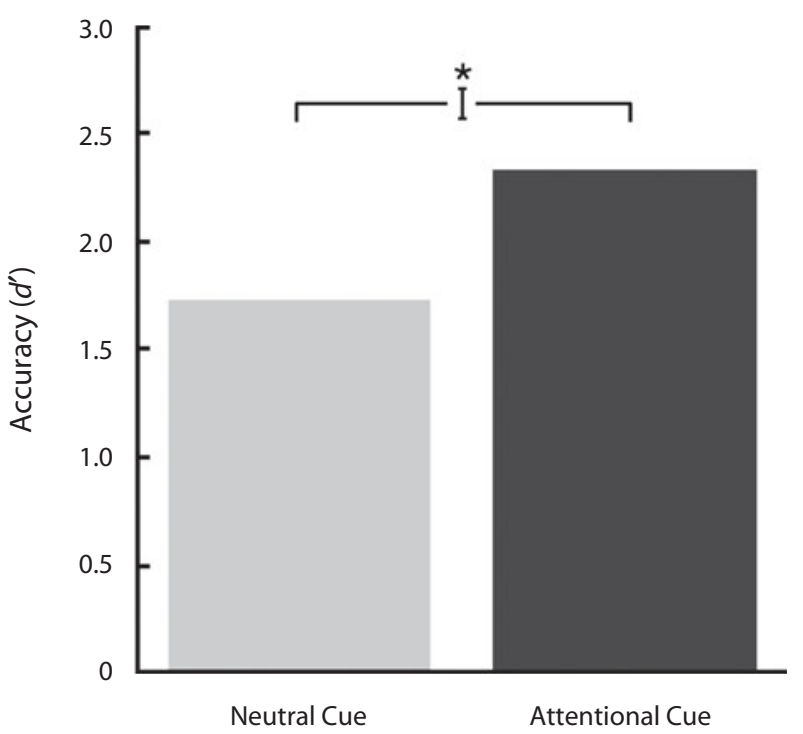

\section{B Control Experiment; $|\mathrm{S}|=\mathbf{5 0 0} \mathrm{msec}$}

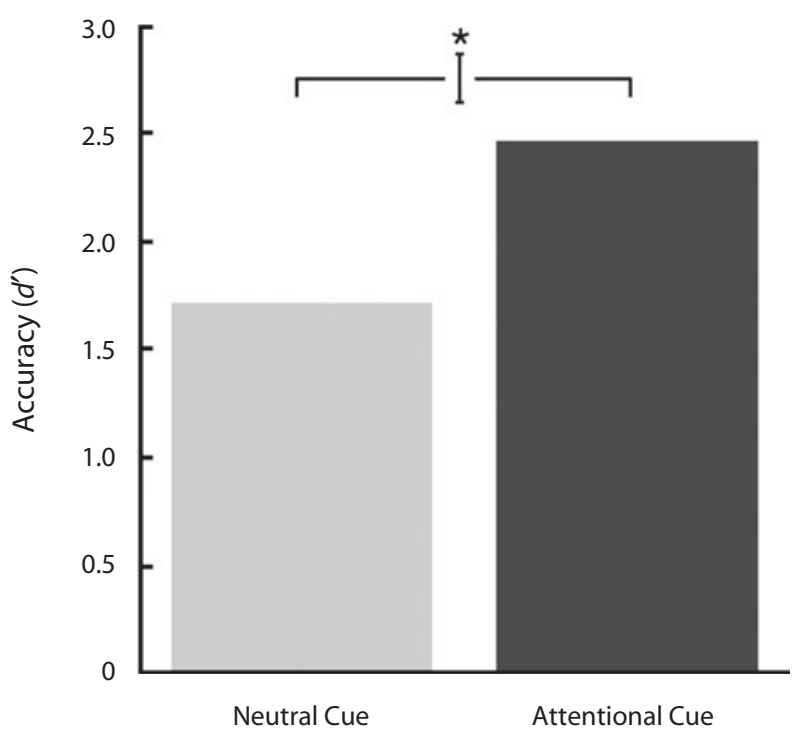

Figure 2. Rapid serial visual presentation (RSVP) performance in the neutral- and attentional-cue conditions for the (A) main and (B) control experiments. RSVP performance is reported in $d^{\prime}$ units. Error bars are within-subjects standard errors of the means, calculated using the method of Loftus and Masson (1994). ISI, interstimulus interval. 
Fits were performed using maximum likelihood estimation, and goodness of fit was evaluated with deviance scores, which are the log-likelihood ratio between a fully saturated, zero residual model and the data model. A score above the critical chi-square value indicates a significant deviation between the fit and the data (Wichmann \& Hill, 2001). In all conditions, for all of the observers and for the averaged data, the psychometric functions represent significant fits to the data, since all of the deviance scores were below the critical chi-square value $\left[\chi^{2}(9,0.95)=16.92\right]$.

Figure $3 \mathrm{~A}$ shows the group-averaged appearance psychometric functions and their Weibull fits. Compared with the neutral condition, the test-cued function shifted to the left, indicating that the observers were more likely to choose the test stimulus as being of a higher spatial frequency when it was cued; the reverse was true when the standard was cued. Another way to illustrate the effect is to fit an individual observer's data and derive shifts in the point of subjective equality (PSE) for the three cuing conditions; group-averaged, normalized PSEs are shown in Figure 3C. Single observer PSEs were normalized by taking the ratio of any one PSE and the average of the test-cued, neutral, and standard-cued PSEs. Cuing the test led to lower PSEs, whereas cuing the standard led to higher PSEs. This pattern of results indicates that cuing a stimulus increased its perceived spatial frequency. A one-way ANOVA showed a significant effect of condition $\left[F(2,14)=35.10, p<.001, \eta_{\mathrm{p}}^{2}=.83\right]$, and post hoc comparisons were significant [test cued vs. neutral: $t(7)=6.47, p<.001, d=2.36$; standard cued vs. neutral: $t(7)=3.46, p<.01, d=2.11]$. The distribution of individual normalized PSEs (Figure 3E) shows that all of the standard-cued PSEs were higher than the neutral PSEs (the points fell above the unity line), and all but one testcued PSE were lower than the neutral PSEs (the points fell below the unity line). Therefore, the effect of attention on perceived spatial frequency is highly consistent across observers.

Control experiment. To rule out response bias as an explanation, we conducted a control experiment in which the ISI between RSVP offset and Gabor onset was lengthened to $500 \mathrm{msec}$. Because the peripheral deployment of attention is effortful and would not help a comparative judgment, we assumed that a lengthened ISI would cause the observers to return to a more neutral attentional state. If the observers simply chose the cued stimulus, the effect should remain strong after $500 \mathrm{msec}$. The effect of attention disappeared in this control experiment. The three psychometric functions were indistinguishable (Figure 3B). The normalized PSEs (Figure 3D) show no effect of cuing $[F(2,14)<1]$. Furthermore, the distribution of single observer normalized PSEs collapsed along the unity line, depicting no clear relation between test cued and standard cued (Figure 3F). These data indicate that the appearance finding in the main experiment is due to the presence of attention and not a bias to respond to the cued side of the space.

\section{Orientation Discrimination}

Main experiment. The orientation discrimination task was contingent on the appearance judgment. Here, we report the discrimination performance when the observers chose the standard $(3.5 \mathrm{cpd})$ stimulus in order to compare performance on the same physical stimulus in one of three conditions: when its location was cued, when neither location was cued (neutral), and when the opposite location was cued (uncued) (Fuller \& Carrasco, 2006; Fuller, Rodriguez, \& Carrasco, 2008; Ling \& Carrasco, 2007; Liu et al., 2009; Liu, Fuller, \& Carrasco, 2006). Figure 4A shows averaged discrimination accuracy. A one-way ANOVA revealed a significant effect of cuing condition in the main experiment $[F(2,14)=10.77, p<$ $\left..001, \eta_{\mathrm{p}}^{2}=.61\right]$. Post hoc paired comparisons indicated that discrimination was significantly higher in the cued than in the neutral condition $[t(7)=2.59, p<.05, d=$ $0.69]$, which in turn was higher than that in the uncued condition $[t(7)=3.52, p<.01, d=0.71]$. These results show that cuing improved orientation discrimination and indicate that attention was deployed to the cued location.

Control experiment. In the control experiment, we predicted that with the lengthened ISI, the effect of cuing would disappear. Figure 4B shows averaged discrimination accuracy. A one-way ANOVA revealed a significant effect of cuing $\left[F(2,14)=6.72, p<.01, \eta_{\mathrm{p}}^{2}=.49\right]$. However, post hoc comparisons indicate that performance did not differ between the uncued and cued conditions $[t(7)=$ $1.58, p=.16]$. Performance in the neutral condition was significantly higher than in the uncued condition $[t(7)=$ $3.36, p<.05, d=0.55$ ] and marginally higher than in the cued condition $[t(7)=2.17, p<.1]$. This finding suggests that with the neutral cue, the observers deployed their attention in a distributed rather than focal manner for the entire trial duration, resulting in better performance. From these data, we can conclude that attention was no longer deployed to the cued location.

\section{DISCUSSION}

In the main and control experiments, we demonstrated that voluntary attention increased perceived spatial frequency and that this effect was not due to response bias. In the main experiment, the voluntary deployment of attention to the cued location improved RSVP performance and orientation discrimination and led to a systematic shift in psychometric functions' PSEs. Perceived spatial frequency was about $0.15 \mathrm{cpd}$ higher than the physical spatial frequency when the test stimulus was cued and about 0.15 cpd lower when the standard stimulus was cued. The extent of this effect was similar in magnitude to that of involuntary attention on perceived spatial frequency ( $\pm 0.18 \mathrm{cpd}$; Gobell \& Carrasco, 2005). In the control experiment, the lengthened ISI extinguished the effect on orientation discrimination and abolished the appearance effect, suggesting that attention was no longer deployed at the cued location. The timing of this decline in the attentional effect is consistent with findings on the dwell time of attention (Horowitz et al., 2009; Theeuwes et al., 2004). The present results demonstrate that the change in appearance is tied to the spatial deployment of attention and not to a simple preference for the previously cued location. 
A Main Experiment; $|\mathrm{S}| \mathbf{=} \mathbf{1 0 0} \mathbf{m s e c}$

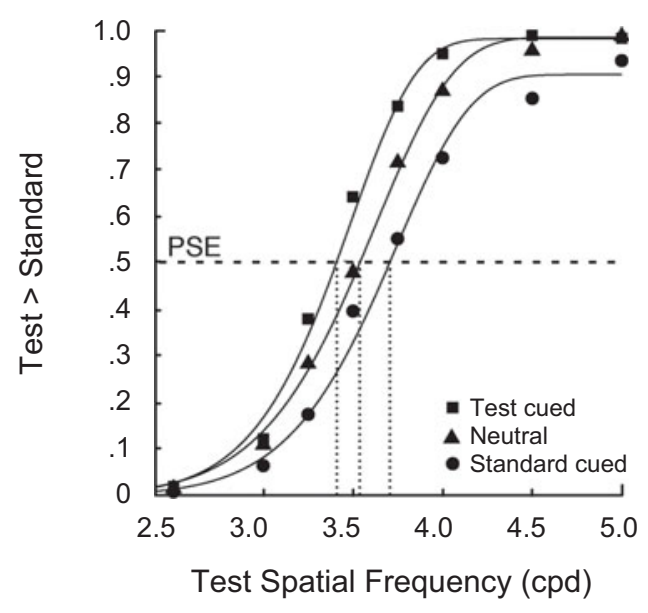

C Main Experiment; $\mid \mathbf{I S} \mathbf{=} \mathbf{1 0 0} \mathrm{msec}$

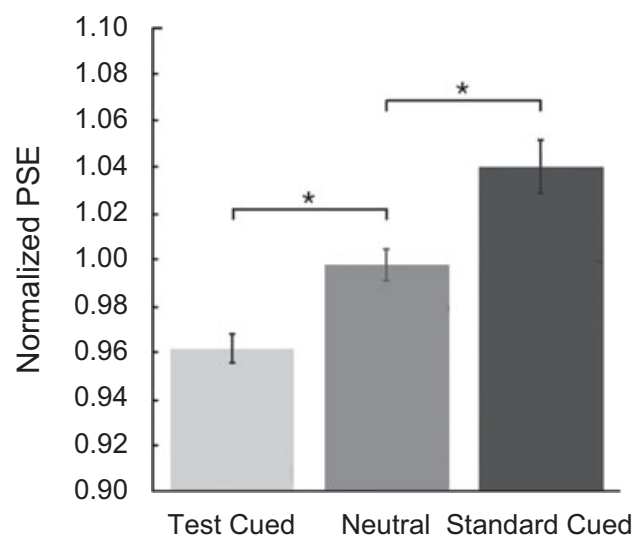

E Main Experiment; $|\mathbf{S}|=100 \mathrm{msec}$

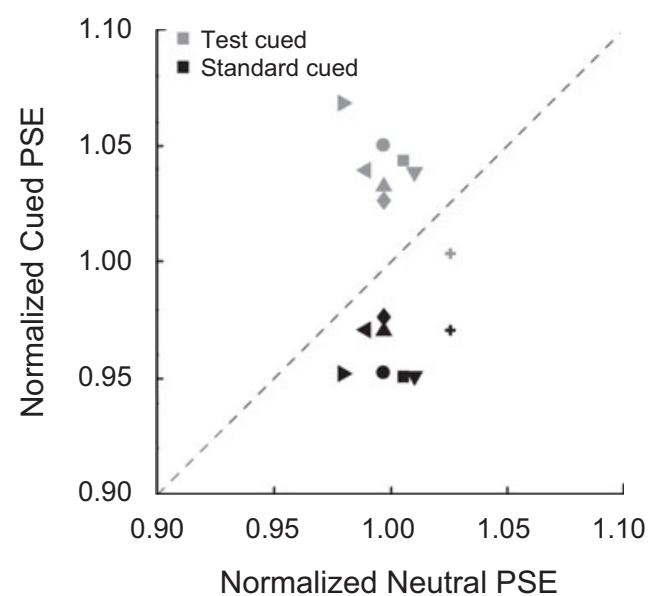

B Control Experiment; ISI $=\mathbf{5 0 0} \mathbf{~ m s e c}$

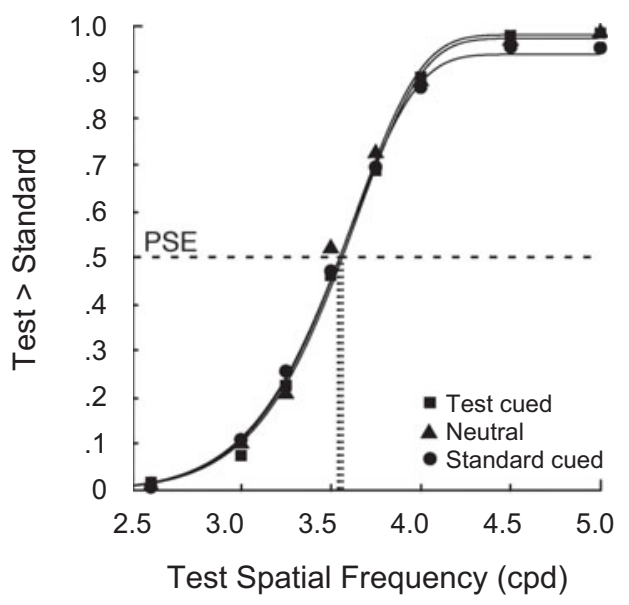

D Control Experiment; $|\mathrm{S}| \mathbf{~} \mathbf{5 0 0} \mathbf{~ m s e c}$

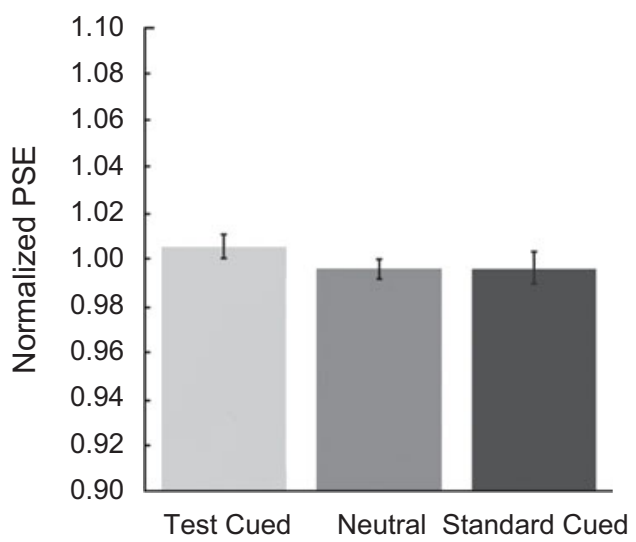

\section{F Control Experiment; $\mid \mathrm{SI} \mathbf{=} \mathbf{5 0 0} \mathbf{~ m s e c}$}

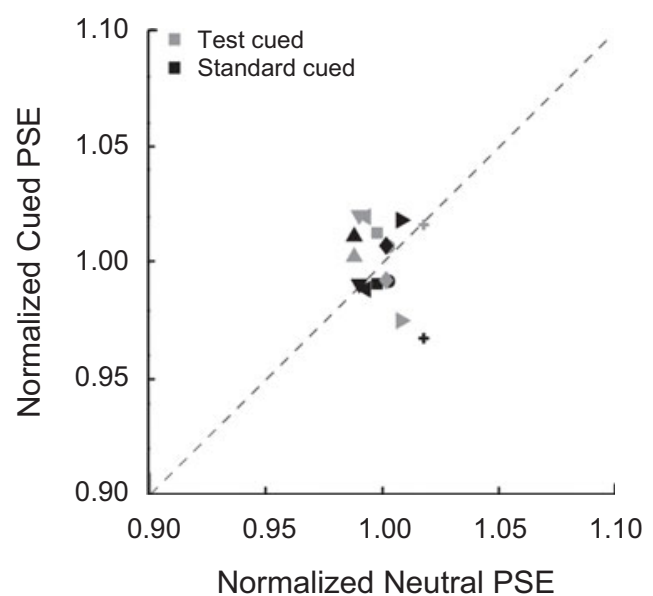

Figure 3. Results for the contrast judgments. The top row presents psychometric functions for the (A) main and (B) control experiments. The graphs show the proportions of trials on which the observers chose the test stimulus to be of higher spatial frequency than the standard stimulus, as a function of the physical spatial frequency of the test stimulus. The middle row presents the normalized values of the point of subjective equality (PSE) for the test stimulus for each of the three cue types in the (C) main and (D) control experiments. Error bars are standard errors of the means, calculated using the method of Loftus and Masson (1994). The bottom row presents scatterplots of individual observers' normalized PSEs in the (E) main and (F) control experiments; each observer's normalized PSEs for test stimuli in the test-cued and standard-cued conditions are plotted as a function of that observer's PSE for the test stimuli in the neutral-cue condition. ISI, interstimulus interval. ${ }^{*} p<.01$. 
A Main Experiment; $\mid \mathbf{S I}=100 \mathrm{msec}$

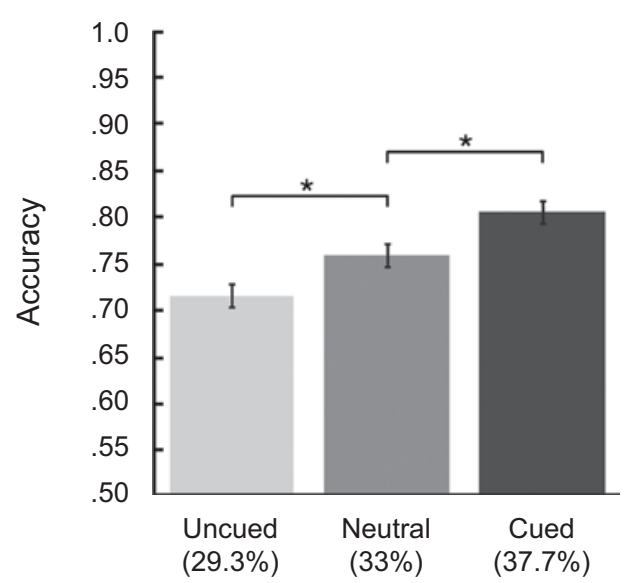

B Control Experiment; ISI $=\mathbf{5 0 0} \mathrm{msec}$

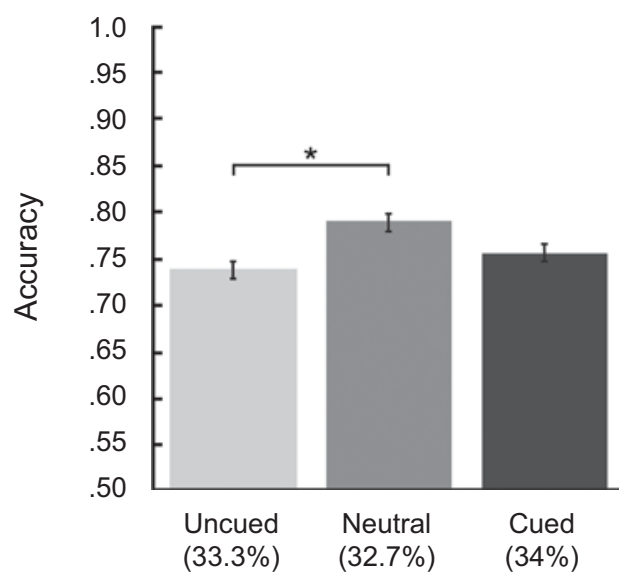

Figure 4. Orientation discrimination performance (accuracy) for trials on which the observers chose the standard stimulus. Performance in the (A) main and (B) control experiments is graphed as a function of cuing condition: cued (i.e., standard cued), uncued (i.e., test cued), or neutral (i.e., neither stimulus cued). Error bars represent standard errors of the means, calculated using the method of Loftus and Masson (1994). The percentage of trials on which the observers selected the standard stimulus in each condition is indicated. ISI, interstimulus interval. ${ }^{*} p<.05$.

Similar to its effects on perceived contrast (Liu et al., 2009), voluntary attention increases perceived spatial frequency. Furthermore, Tse (2005) demonstrated that voluntary attention and perceptual grouping mechanisms modulate perceived brightness by changing the perceived structure of the visual scene. The present study, along with those by Liu et al. (2009) and Tse (2005), provides evidence that voluntary attention alters perception along a number of basic visual dimensions, indicating that it acts at an early stage of visual processing.

A previous study suggested that voluntary attention does not affect perceived spatial frequency but simply reduces response variability (Prinzmetal et al., 1998). However, in that study, the location of spatial attention was not manipulated; instead, a dual-task procedure was used, and the difficulty of the primary letter identification task (simultaneous vs. sequential presentation) was varied to manipulate attentional deployment in the secondary appearance task. Furthermore, there was no independent measurement ensuring that attention had been deployed to the correct location, which is necessary to confirm the successful allocation of attention. For example, in a study in which attention did not affect perceived hue, there was an effect on tilt discrimination at the attended location, confirming that attention was deployed successfully but that it did not affect appearance (Fuller \& Carrasco, 2006). Without control or measurement of spatial attention, Prinzmetal et al.'s (1998) results are inconclusive (Carrasco, Ling, \& Read, 2004; Treue, 2004). Given that in the present study we explicitly manipulated and measured the spatial deployment of attention (using the RSVP task and orientation discrimination task, respectively), we can conclude that voluntary covert attention does alter perceived spatial frequency.

Provided that attention enhances contrast sensitivity and perceived contrast (for a review, see Carrasco, 2006), could it be argued that the effects of attention on the perceived spatial frequency might be mediated by an increase in perceived contrast? This possibility is unlikely, because increasing the physical contrast of the test stimulus above that of a $50 \%$ contrast standard stimulus makes observers more likely to report the test stimulus as lower in spatial frequency than the standard (Georgeson, 1985; Gobell \& Carrasco, 2005). These findings indicate that an increase in perceived contrast alone would decrease perceived spatial frequency. Thus, if anything, the increase in perceived spatial frequency reported here might be underestimated, since increases in perceived contrast may act to lower the perceived spatial frequency.

In both the main experiment and the control experiment, we used an RSVP task to manipulate voluntary attention. The observers were exposed for $1.2 \mathrm{sec}$ to high-contrast, serially presented letter stimuli. Could it be argued that this RSVP is visually akin to flicker and thus leads to flicker adaptation? Moreover, given that attention strengthens adaptation (e.g., Ling \& Carrasco, 2006b), could it be argued that attention intensifies such hypothetical flicker adaptation at the cued location? First, flicker adaptation seems unlikely in the present study, because previous experimenters have utilized gratings with fixed spatial and temporal frequencies or spatially overlapping discs to induce flicker adaptation (e.g., Anstis, 1996; Parker, 1981; Smith, 1971; for a review, see Kohn, 2007). In contrast, the letters used in the RSVP task have a broad frequency spectrum, their sequential presentation does not ensure a continuous cyclical dark:light oscillation, and the Gabor appears stationary for $40 \mathrm{msec}$ either 100 or $500 \mathrm{msec}$ after the RSVP offset in the main or control experiment, respectively. Second, although to our knowledge there is no empirical demonstration that attention enhances flicker adaptation, this hypothetical effect is plausible, 
given that attention enhances adaptation in some domains (e.g., Ling \& Carrasco, 2006b; Montaser-Kouhsari \& Rajimehr, 2004). The duration of adaptation aftereffects is proportional to the adapting time on a log-log scale (e.g., Magnussen \& Johnsen, 1986), and the effects are still strong at $50 \%$ of the adaptation duration (Snippe, Poot, \& van Hateren, 2004; Snippe \& van Hateren, 2003). Thus, in the present study, the effect of adaptation would have lasted long enough to alter the perceived frequency of the Gabor in both the main experiment and the control experiment (the Gabor stimuli disappeared 140 and $540 \mathrm{msec}$ after the RSVP offset, respectively; Figure 1). Nevertheless, at the cued location, perceived spatial frequency was higher in the main experiment but not in the control experiment (Figure 3). Hence, these findings cannot be explained by an adaptation effect but are consistent with the dynamics of voluntary attention discussed above.

\section{Spatial Resolution and Perceived Frequency}

The finding that attention increases perceived spatial frequency is consistent with studies showing that spatial resolution is enhanced by both involuntary and voluntary attention (Carrasco, Loula, \& Ho, 2006; Carrasco, Williams, \& Yeshurun, 2002; Golla, Ignashchenkova, Haarmeier, \& Thier, 2004; Montagna et al., 2009; Talgar \& Carrasco, 2002; Yeshurun \& Carrasco, 1998, 1999, 2000; Yeshurun et al., 2008). Others have reported that voluntary attention reduces orientation thresholds measured with flanker stimuli to levels seen with no flankers present, and they have thus proposed that attention reduces the scale over which an image is analyzed (Morgan, Ward, \& Castet, 1998). In another key finding, when the target location was cued, perceived line length was reduced (Tsal \& Shalev, 1996). These authors concluded that smaller receptive fields mediate the effect of involuntary attention, increasing spatial resolution. In a subsequent study, they ruled out spatial interactions between the cue and the target, as well as cue salience, as factors that could interact with line-length judgments (Tsal, Shalev, \& Zakay, 2005). Furthermore, consistent with the idea that attention increases spatial resolution, cuing a peripheral line with a small gap enables observers to detect smaller gaps (Shalev \& Tsal, 2002).

The present findings fit well with the majority of the literature on attention and spatial resolution. However, according to the flexibility of voluntary attention on performance studies (e.g., Giordano et al., 2009; Yeshurun et al., 2008), the effects of involuntary attention need not necessarily parallel those of voluntary attention on appearance. Here, we found that for the tested frequency range, the effect of voluntary attention on perceived frequency is consistent with that of involuntary attention (Gobell \& Carrasco, 2005). We can relate the present behavioral findings on voluntary attention with neurophysiological studies in which the effects of voluntary attention on spatial resolution have been investigated (e.g., Carrasco \& Yeshurun, 2009).

\section{Mechanisms of Enhanced Resolution}

Recent neurophysiological studies have offered insight into the potential mechanisms of enhanced resolution.
These findings have indicated shifting and shrinking in receptive fields with endogenous attention in areas MT and LIP (Anton-Erxleben et al., 2009; Kusunoki \& Goldberg, 2003; Womelsdorf et al., 2006). Specifically, if both a preferred and a nonpreferred stimulus are present in a neuron's receptive field, that cell's responsiveness depends on the attentional state of the animal. Attending to the preferred stimulus increases the cell's firing rate, whereas attending to the nonpreferred stimulus attenuates it. This finding suggests that attention shifts and/or constricts the receptive field of the cell at the attended location (as was suggested by Moran \& Desimone, 1985, and Reynolds \& Desimone, 1999, and shown by Anton-Erxleben et al., 2009, and Womelsdorf et al., 2006). Such shifting and shrinking provide a possible neural mechanism for the higher resolution analyses that have been reported in the behavioral literature. These findings all suggest that voluntary attention could act by allowing for a more finegrained analysis of the attended area. However, a finergrained representation would not necessarily lead to the percept of higher spatial frequency, and it is not the only way to enhance spatial resolution.

Attention could also enhance spatial resolution by reweighting the population response in favor of higher spatial frequency receptors (Balz \& Hock, 1997; Carrasco, Loula, \& Ho, 2006). This sort of mechanism and its impact on phenomenology are illustrated in Figure 5. In the absence of attention, a stimulus of a particular spatial frequency (indicated by the orange vertical bar) will produce a particular pattern of differential activity in the population of distinct receptors sensitive to spatial frequency (see the subset of channels in red, blue, and green). By shifting sensitivity to higher spatial frequencies, attention increases the sensitivity of those channels, resulting in a different activity pattern for this same stimulus (Carrasco, Loula, \& Ho, 2006; Yeshurun \& Carrasco, 2000). This shift of the population response with attention would result in an activity pattern similar to the activity pattern observed for a stimulus of higher spatial frequency in the absence of attention, giving rise to the phenomenological experience of a higher spatial frequency stimulus. Whereas the data presented here suggest the reweighting mechanism as the likely source of perceptual enhancement, these data are not inconsistent with shifting and shrinking of receptive fields, since the two mechanisms are not mutually exclusive.

\section{Ruling Out Cue and Response Biases}

In appearance studies, it is always important to identify and rule out potential cue and response biases. A cue bias may occur when uncertainty about the task leads the observer to select the cued side of the space as his or her answer, whereas a response bias may lead the observer to conclude that the cued stimulus is more relevant or salient. Many controls have been devised to address such biases and have consistently demonstrated that the effects of attention on appearance are tied to early changes in the perceptual quality of the stimulus (for a review, see Carrasco, 2009a).

In the control experiment, we utilized a lengthened ISI to rule out a bias explanation of the findings in the main 
Channels at Baseline

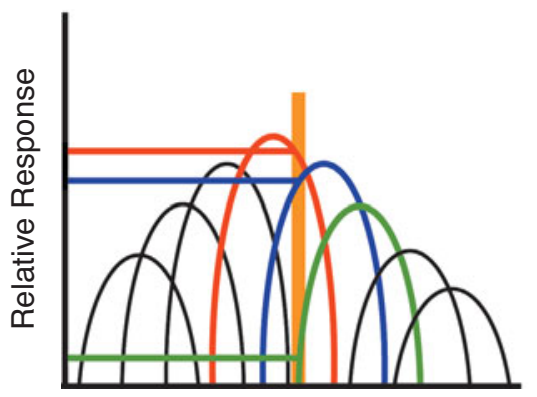

Spatial Frequency (cpd)
Channels With Attention

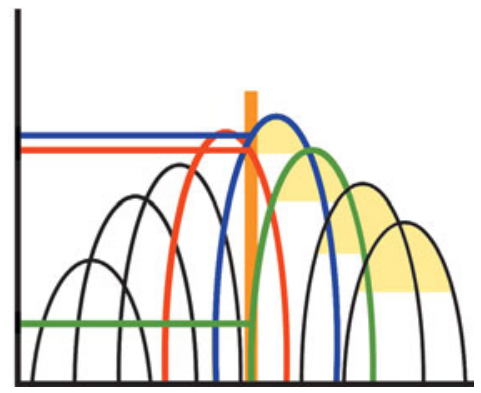

$\bigcap$

Spatial frequency channels

\begin{abstract}
Figure 5. Illustration of how attention might produce a change in apparent spatial frequency via a shift in sensitivity to higher spatial frequencies. The left panel schematically illustrates the baseline sensitivity of different channels, and the right panel illustrates increased sensitivity for the channels of higher spatial frequency with attention (illustrated in light orange). The vertical bar illustrates a stimulus of a particular frequency, and the horizontal lines indicate the response of three illustrative channels.
\end{abstract}

experiment. Increasing the ISI, when the observers knew that the cue carried no information about the post-ISI task, gave the observers the opportunity to redeploy attention. Studies of involuntary attention have shown that the precue alters appearance at short ISIs $(100-130 \mathrm{msec})$ when the effects of involuntary attention peak, but not at long ISIs $(500 \mathrm{msec})$, because the effects of involuntary attention have already decayed (Anton-Erxleben, Henrich, \& Treue, 2007; Carrasco, Ling, \& Read, 2004; Fuller et al., 2008; Liu et al., 2006; Turatto, Vescovi, \& Valsecchi, 2007). In both the involuntary paradigm and the voluntary paradigm, lengthening the ISI provides the time necessary for either the decay or the redeployment of attention, respectively, abolishing the effect. The clear link between the appearance effect and the continued presence of attention indicates that this effect is dependent on the perceptual modulation of the stimulus representation and not a cognitive prioritization that gives rise to a response bias.

Previous studies concerning attention and appearance have ruled out bias in other ways, such as reversing the task. Reversed instructions were first used in a study on prior entry and bias in temporal order judgments (Shore, Spence, \& Klein, 2001). Shore et al. found that observers chose the cued stimulus when asked either "Which stimulus came first?" or "Which stimulus came second?" They concluded that their finding of prior entry with cuing was partially due to bias that had to be corrected for. Crucially, in studies of the effect of voluntary and involuntary attention on appearance, when reversing the task instructions (e.g., choose the stimulus of lower contrast), observers do not choose the cued stimulus (Anton-Erxleben et al., 2007; Carrasco, Ling, \& Read, 2004; Liu et al., 2009; Montagna \& Carrasco, 2006). Had there been a bias, as in Shore et al.'s study, observers would have reported that the cued stimulus had lower contrast.
It is also important to empirically verify that the attention effect is due to a change in perception and not to an increased likelihood of responding to the cued location. When using near-threshold stimuli, a cue bias exists (Carrasco et al., 2008; Prinzmetal, Long, \& Leonhardt, 2008). However, with suprathreshold stimuli, attention increases perceived contrast, and the attention effects are not due to a cue bias (Carrasco et al., 2008; Carrasco, Ling, \& Read, 2004; Ling \& Carrasco, 2007). When observers are capable of localizing and resolving the stimuli, as in the present experiments, they perform the appearance judgment, whereas when there is location uncertainty, their responses are driven by the cue location (Carrasco et al., 2008; Prinzmetal et al., 2008).

Other findings cannot be explained by a bias account. For instance, the effect of attention on appearance varies at different locations in the visual field: The effect is larger on the lower than on the upper vertical meridian. However, a cue bias would predict the opposite, since it is supposed to be more pronounced with low-contrast stimuli, and contrast sensitivity is lower in the upper vertical meridian (Fuller et al., 2008). Furthermore, attention alters perceived saturation but not perceived hue, notwithstanding the fact that attention improves performance with stimuli mediated by both dimensions (Fuller \& Carrasco, 2006).

\section{Perceptual Versus Decisional Effects on Appearance}

Some authors have claimed that comparative paradigms (e.g., greater than), like the one used in the present study, cannot distinguish between changes in PSE due to salience and actual changes in perception, whereas equality paradigms can make this distinction (Schneider \& Komlos, 2008; Valsecchi, Vescovi, \& Turatto, 2010). Schneider and Komlos's null results with the equality paradigm and their 
positive result with the comparative paradigm have led them to argue that attention makes stimuli more salient, biasing decisions rather than altering perception. These conclusions are unwarranted for three reasons: (1) the inconsistent theoretical relation among salience, perception, and attention; (2) issues with criteria in the equality paradigm; and (3) the difficulty in interpreting their null results.

Schneider and Komlos (2008) argued that attention alters the salience of stimuli without changing the observers' phenomenological experience. In this view, attention operates via a prioritization mechanism that makes stimuli more readily available for processing but does not change their perceptual quality. Schneider and Komlos claimed that in all studies of attention and appearance, "attention is reported to modify the appearance of stimuli to increase their salience" (p. 9). For example, higher contrast is more salient, but a more salient stimulus is not necessarily higher in contrast. Essentially, when an experimenter finds that attention increases perceived contrast or perceived spatial frequency, the observers have simply been selecting the more salient stimulus, rather than the stimulus that actually appears higher on the dimension of interest. However, the relation between salience in the spatial frequency domain and salience in the contrast domain is not that simple. Higher spatial frequency stimuli appear lower in contrast, and decreasing stimulus contrast makes spatial frequency appear higher (Georgeson, 1985; Gobell \& Carrasco, 2005). Contrary to the predictions of a salience account, the shift in spatial frequency observed in the present study is in the direction of decreased salience at the attended location.

Schneider and Komlos (2008) argued that the equality paradigm is superior to the comparative paradigm because shifts in PSE are unrelated to shifts in criteria. They contrast this with comparative judgments, in which they argue that shifts in criterion and central tendency are degenerate (Schneider \& Bavelier, 2003; Schneider \& Komlos, 2008; Valsecchi et al., 2010). For the PSE and the criteria to be independent in the equality paradigm, the two criteria must be symmetric. Indeed, symmetry must be verified empirically, because observers often use asymmetric criteria in equality judgment tasks (Petrov, 2009). Furthermore, in an experiment designed to test sensitivity and bias in the equality paradigm, Valsecchi et al. found that the standard deviation, amplitude, and mean of their psychometric functions were significantly correlated, indicating a violation of independence. This nonindependence of the parameters undermines the main justification for using the equality paradigm.

Beyond the limitations of the equality paradigm, further methodological problems limit the interpretation of Schneider and Komlos's (2008) results. Because their experiment had no neutral condition, there was no baseline from which to assess a shift in PSE. Therefore, their observed differences in PSE between the two paradigms could be due to different baselines. This is likely, because observers are generally less accurate in equality paradigms than they are in comparative paradigms (Fetterman, Dreyfus, \& Stubbs, 1996). Widely differing baselines have also been reported between the two paradigms (Valsecchi et al., 2010), suggesting that the assumption of veridicality by Schneider and Komlos is unwarranted. Furthermore, fits to the data are poorer and parameter estimates are more variable in the equality paradigm (Valsecchi et al., 2010). Therefore, interpreting a null result from that paradigm is problematic, because noise and inaccuracy could obscure an attentional effect.

Finally, in a recent study in our lab, the strengths and weakness of the equality paradigm were assessed, and we sought to determine whether that paradigm could detect changes in both physical and perceived contrast (AntonErxleben, Abrams, \& Carrasco, 2010). In Anton-Erxleben et al. (2010), we utilized physical contrast increments to assess sensitivity and bias in both the equality paradigm and the comparative paradigm and used a cuing paradigm to examine the effects of attention. We found that both paradigms could detect changes in physical contrast, although the PSE estimates in the equality paradigm were less accurate and much noisier than those in the comparative paradigm, which is similar to the findings with speed of Valsecchi et al. (2010). Notwithstanding these differences, attention increased perceived contrast in both experiments, but the effect was smaller with the equality than with the comparative paradigm.

To summarize, at the present time, there is no reason to conclude that the equality paradigm is a better method than the comparative paradigm for the study of appearance. The correlation of parameter estimates in the equality paradigm suggests that changes in criteria do affect the central tendency. Because the independence of parameters is the justification for using the paradigm, the superiority of the equality paradigm in studies of appearance can be rejected. Given the noise and inaccuracy in the equality paradigm, as well as the possibility of asymmetric criteria (e.g., Petrov, 2009), interpreting a null result from that paradigm as evidence against a change in appearance is unsound. The present study demonstrates that attention leads to a reliable shift in PSEs, that the shift in PSEs is in the direction opposite to that predicted by a salience account, and, thus, that attention increases perceived spatial frequency.

\section{Conclusion}

The present findings advance our understanding of the effects of attention on the phenomenology of spatial vision by showing that voluntary attention increases perceived spatial frequency. Involuntary attention enhances perceived contrast (Carrasco et al., 2008; Carrasco, Ling, $\&$ Read, 2004) and spatial frequency (Gobell \& Carrasco, 2005 ), and voluntary attention enhances perceived contrast (Liu et al., 2009). Because of the present findings, we conclude that attention affects early visual processing, boosting signal strength and spatial resolution. This early modulation alters the phenomenological experience of our visual environment, which is consistent with behavioral findings that attention increases performance on tasks mediated by contrast and spatial resolution (for reviews, see Carrasco, 2006; Carrasco \& Yeshurun, 2009), as well as with physiological effects of attention on the 
BOLD response in early visual cortex (e.g., Fischer \& Whitney, 2009; Liu, Pestilli, \& Carrasco, 2005). Finally, recent electrophysiological research on cross-modal attention and appearance demonstrates that the time course, location, and amplitude of the evoked response to an attended stimulus is indicative of a perceptual modulation, rather than of a decisional effect (Störmer, McDonald, \& Hillyard, 2009; see also Carrasco, 2009b). In summary, attention modulates early perceptual mechanisms, which fundamentally alter the percept and lead to changes in appearance.

\section{AUTHOR NOTE}

This research was supported by Grants NIH RO1 EY016200 to M.C. and NIH T32 EY007136 to NYU. We thank Katharina Anton-Erxleben and the rest of the Carrasco lab members for their valuable comments. Correspondence concerning thise article should be addressed to M. Carrasco, Department of Psychology, New York University, 6 Washington Place, 8th Floor, New York, NY 10003 (e-mail: marisa.carrasco@nyu.edu).

\section{REFERENCES}

Anstis, S. (1996). Adaptation to peripheral flicker. Vision Research, 36, 3479-3485.

Anton-Erxleben, K., Abrams, J., \& Carrasco, M. (2010, May). Attention does alter apparent contrast: Evaluating comparative and equality judgments. Poster session presented at the 10th Annual Meeting of the Vision Sciences Society, Naples, Florida.

Anton-Erxleben, K., Henrich, C., \& Treue, S. (2007). Attention changes perceived size of moving visual patterns. Journal of Vision, 7(11, Art. 5), 1-9.

Anton-Erxleben, K., Stephan, V. M., \& Treue, S. (2009). Attention reshapes center-surround receptive-field structure in macaque cortical area MT. Cerebral Cortex, 19, 2466-2478.

BaLz, G. W., \& Hock, H. S. (1997). The effect of attentional spread on spatial resolution. Vision Research, 37, 1499-1510.

CARRASCO, M. (2006). Covert attention increases contrast sensitivity: Psychophysical, neurophysiological, and neuroimaging studies. In S. Martinez-Conde, S. L. Macknik, L. M. Martinez, J. M. Alonso, \& P. U. Tse (Eds.), Visual perception: Part 1. Fundamentals of vision (pp. 33-70). Amsterdam: Elsevier.

CARrasco, M. (2009a). Attention: Psychophysical approaches. In T. Bayne, A. Cleeremans, \& P. Wilken (Eds.), The Oxford companion to consciousness (pp. 78-84). Oxford: Oxford University Press.

CARrasco, M. (2009b). Cross-modal attention enhances perceived contrast. Proceedings of the National Academy of Sciences, 106, 2203922040.

Carrasco, M., Fuller, S., \& Ling, S. (2008). Transient attention does increase perceived contrast of suprathreshold stimuli: A reply to Prinzmetal, Long, and Leonhardt (2008). Perception \& Psychophysics, 70, 1151-1164.

Carrasco, M., Giordano, A. M., \& McElree, B. (2004). Temporal performance fields: Visual and attentional factors. Vision Research, 44, 1351-1365.

Carrasco, M., Giordano, A. M., \& McElree, B. (2006). Attention speeds processing across eccentricity: Feature and conjunction searches. Vision Research, 46, 2028-2040.

Carrasco, M., Ling, S., \& Read, S. (2004). Attention alters appearance. Nature Neuroscience, 7, 308-313.

Carrasco, M., Loula, F., \& Ho, Y.-X. (2006). How attention enhances spatial resolution: Evidence from selective adaptation to spatial frequency. Perception \& Psychophysics, 68, 1004-1012.

Carrasco, M., \& McElree, B. (2001). Covert attention accelerates the rate of visual information processing. Proceedings of the National Academy of Sciences, 98, 5363-5367.

Carrasco, M., Williams, P. E., \& Yeshurun, Y. (2002). Covert attention increases spatial resolution with or without masks: Support for signal enhancement. Journal of Vision, 2(6, Art. 4), 467-479.

Carrasco, M., \& Yeshurun, Y. (2009). Covert attention effects on spa- tial resolution. In S. Narayanan (Ed.), Attention (pp. 65-86). Amsterdam: Elsevier.

Fetterman, J. G., Dreyfus, L. R., \& Stubbs, D. A. (1996). Judging relative duration: The role of rule and instructional variables. Journal of Experimental Psychology: Animal Behavior Processes, 22, 350-361.

Fischer, J., \& WhitNey, D. (2009). Attention narrows position tuning of population responses in V1. Current Biology, 19, 1356-1361.

Fuller, S., \& Carrasco, M. (2006). Exogenous attention and color perception: Performance and appearance of saturation and hue. Vision Research, 46, 4032-4047.

Fuller, S., PARK, Y., \& Carrasco, M. (2009). Cue contrast modulates the effects of exogenous attention on appearance. Vision Research, 49, 1825-1837.

Fuller, S., Rodriguez, R. Z., \& Carrasco, M. (2008). Apparent contrast differs across the vertical meridian: Visual and attentional factors. Journal of Vision, 8(1, Art. 16), 1-16.

Georgeson, M. A. (1985). Apparent spatial frequency and contrast of gratings: Separate effects of contrast and duration. Vision Research, 25, 1721-1727.

Giordano, A. M., McElree, B., \& Carrasco, M. (2009). On the automaticity and flexibility of covert attention: A speed-accuracy tradeoff analysis. Journal of Vision, 9(3, Art. 30), 1-10.

Gobell, J., \& CARRASCO, M. (2005). Attention alters the appearance of spatial frequency and gap size. Psychological Science, 16, 644-651.

Golla, H., Ignashchenkova, A., HaArmeier, T., \& Thier, P. (2004). Improvement of visual acuity by spatial cuing: A comparative study in human and nonhuman primates. Vision Research, 44, 1589-1600.

Horowitz, T. S., Wolfe, J. M., Alvarez, G. A., Cohen, M. A., \& Kuzmova, Y. I. (2009). The speed of free will. Quarterly Journal of Experimental Psychology, 62, 2262-2288.

KoHN, A. (2007). Visual adaptation: Physiology, mechanisms, and functional benefits. Journal of Neurophysiology, 97, 3155-3164.

KusunOKI, M., \& GoldBERG, M. E. (2003). The time course of perisaccadic receptive field shifts in the lateral intraparietal area of the monkey. Journal of Neurophysiology, 89, 1519-1527.

LenNiE, P. (2003). The cost of cortical computation. Current Biology, 13, 493-497.

Ling, S., \& Carrasco, M. (2006a). Sustained and transient covert attention enhance the signal via different contrast response functions. Vision Research, 46, 1210-1220.

Ling, S., \& Carrasco, M. (2006b). When sustained attention impairs perception. Nature Neuroscience, 9, 1243-1245.

Ling, S., \& CARRASCO, M. (2007). Transient covert attention does alter appearance: A reply to Schneider (2006). Perception \& Psychophysics, 69, 1051-1058.

Liu, T., Abrams, J., \& Carrasco, M. (2009). Voluntary attention enhances contrast appearance. Psychological Science, 20, 354-362.

Liu, T., Fuller, S., \& Carrasco, M. (2006). Attention alters the appearance of motion coherence. Psychonomic Bulletin \& Review, 13, 1091-1096.

LiU, T., Pestilli, F., \& Carrasco, M. (2005). Transient attention enhances perceptual performance and fMRI response in human visual cortex. Neuron, 45, 469-477.

LoFtus, G. R., \& MASSON, M. E. J. (1994). Using confidence intervals in within-subject designs. Psychonomic Bulletin \& Review, 1, 476-490.

Lu, Z.-L., \& Dosher, B. A. (2000). Spatial attention: Different mechanisms for central and peripheral temporal precues? Journal of Experimental Psychology: Human Perception \& Performance, 26, 15341548.

LUCK, S. J. (2004). Understanding awareness: One step closer. Nature Neuroscience, 7, 208-209.

Magnussen, S., \& Johnsen, T. (1986). Temporal aspects of spatial adaptation: A study of the tilt aftereffect. Vision Research, 26, 661-672.

Montagna, B., \& CARrasco, M. (2006). Transient covert attention and the perceived rate of flicker. Journal of Vision, 6(9, Art. 8), 955-965.

Montagna, B., Pestilli, F., \& Carrasco, M. (2009). Attention trades off spatial acuity. Vision Research, 49, 735-745.

Montaser-Kouhsari, L., \& RaJimehr, R. (2004). Attentional modulation of adaptation to illusory lines. Journal of Vision, 4(6, Art. 3), 434-444.

Moran, J., \& Desimone, R. (1985). Selective attention gates visual processing in the extrastriate cortex. Science, 229, 782-784. 
Morgan, M. J., Ward, R. M., \& CASTET, E. (1998). Visual search for a tilted target: Tests of spatial uncertainty models. Quarterly Journal of Experimental Psychology, 51A, 347-370.

Müller, H. J., \& RABbitT, P. M. (1989). Reflexive and voluntary orienting of visual attention: Time course of activation and resistance to interruption. Journal of Experimental Psychology: Human Perception \& Performance, 15, 315-330.

Nakayama, K., \& Mackeben, M. (1989). Sustained and transient components of focal visual attention. Vision Research, 29, 1631-1647.

PARKER, A. (1981). Shifts in perceived periodicity induced by temporal modulation and their influence on the spatial frequency tuning of two aftereffects. Vision Research, 21, 1739-1747.

Pestilli, F., \& Carrasco, M. (2005). Attention enhances contrast sensitivity at cued and impairs it at uncued locations. Vision Research, 45, 1867-1875.

Pestilli, F., Ling, S., \& Carrasco, M. (2009). A population-coding model of attention's influence on contrast response: Estimating neural effects from psychophysical data. Vision Research, 49, 1144-1153.

Pestilli, F., Viera, G., \& Carrasco, M. (2007). How do attention and adaptation affect contrast sensitivity? Journal of Vision, 7(7, Art. 9), $1-12$.

Petrov, A. A. (2009). Symmetry-based methodology for decision-rule identification in same-different experiments. Psychonomic Bulletin \& Review, 16, 1011-1025.

Posner, M. I. (1980). Orienting of attention. Quarterly Journal of Experimental Psychology, 32, 3-25.

Prinzmetal, W., Amiri, H., Allen, K., \& Edwards, T. (1998). Phenomenology of attention: 1 . Color, location, orientation, and spatial frequency. Journal of Experimental Psychology: Human Perception \& Performance, 24, 261-282.

Prinzmetal, W., Long, V., \& Leonhardt, J. (2008). Involuntary attention and brightness contrast. Perception \& Psychophysics, 70, 11391150

Prinzmetal, W., Nwachuku, I., Bodanski, L., Blumenfeld, L., \& ShimizU, N. (1997). The phenomenology of attention: 2. Brightness and contrast. Consciousness \& Cognition, 6, 372-412.

ReYnolds, J. H., \& Desimone, R. (1999). The role of neural mechanisms of attention in solving the binding problem. Neuron, 24, 19-29.

Schneider, K. A., \& BAVElier, D. (2003). Components of visual prior entry. Cognitive Psychology, 47, 333-366.

Schneider, K. A., \& Komlos, M. (2008). Attention biases decisions but does not alter appearance. Journal of Vision, 8(15, Art. 3), 1-10.

Shalev, L., \& Tsal, Y. (2002). Detecting gaps with and without attention: Further evidence for attentional receptive fields. European Journal of Cognitive Psychology, 14, 3-26.

Shore, D. I., Spence, C., \& Klein, R. M. (2001). Visual prior entry. Psychological Science, 12, 205-212.

SмiтH, R. A. (1971). Studies of temporal frequency adaptation in visual contrast sensitivity. Journal of Physiology, 216, 531-552.

Snippe, H. P., Poot, L., \& van Hateren, J. H. (2004). Asymmetric dynamics of adaptation after onset and offset of flicker. Journal of Vision, 4(1, Art. 1), 1-12

Snippe, H. P., \& VAN Hateren, J. H. (2003). Recovery from contrast adaptation matches ideal-observer predictions. Journal of the Optical Society of America, 20, 1321-1330.

Störmer, V. S., McDonald, J. J., \& Hillyard, S. A. (2009). Crossmodal cuing of attention alters appearance and early cortical processing of visual stimuli. Proceedings of the National Academy of Sciences, 106, 22456-22461.

TAlgar, C. P., \& CARRAsco, M. (2002). Vertical meridian asymmetry in spatial resolution: visual and attentional factors. Psychonomic Bulletin \& Review, 9, 714-722.

Theeuwes, J., Godijn, R., \& Pratt, J. (2004). A new estimation of the duration of attentional dwell time. Psychonomic Bulletin \& Review, 11, 60-64.

Treue, S. (2004). Perceptual enhancement of contrast by attention. Trends in Cognitive Sciences, 8, 435-437.

Tsal, Y., \& Shalev, L. (1996). Inattention magnifies perceived length: The attentional receptive field hypothesis. Journal of Experimental Psychology: Human Perception \& Performance, 22, 233-243.

Tsal, Y., ShaleV, L., \& ZaKaY, D. (2005). The lengthening effect revisited: A reply to Prinzmetal and Wilson (1997) and Masin (1999). Psychonomic Bulletin \& Review, 12, 185-190.

TsE, P. U. (2005). Voluntary attention modulates the brightness of overlapping transparent surfaces. Vision Research, 45, 1095-1098.

Turatto, M., Vescovi, M., \& Valsecchi, M. (2007). Attention makes moving objects be perceived to move faster. Vision Research, 47, 166178.

Valsecchi, M., Vescovi, M., \& Turatto, M. (2010). Are the effects of attention on speed judgments genuinely perceptual? Attention, Perception, \& Psychophysics, 72, 637-650.

Wichmann, F. A., \& Hill, N. J. (2001). The psychometric function: I. Fitting, sampling, and goodness of fit. Perception \& Psychophysics, 63, 1293-1313.

Womelsdorf, T., Anton-Erxleben, K., Pieper, F., \& Treue, S. (2006). Dynamic shifts of visual receptive fields in cortical area MT by spatial attention. Nature Neuroscience, 9, 1156-1160.

YANTIS, S., \& Jonides, J. (1990). Abrupt visual onsets and selective attention: Voluntary versus automatic allocation. Journal of Experimental Psychology: Human Perception \& Performance, 16, 121 134.

Yeshurun, Y., \& CARrasco, M. (1998). Attention improves or impairs visual performance by enhancing spatial resolution. Nature, 396, 72-75.

YeSHURUN, Y., \& CARRASCO, M. (1999). Spatial attention improves performance in spatial resolution tasks. Vision Research, 39, 293-306.

Yeshurun, Y., \& CARRASCO, M. (2000). The locus of attentional effects in texture segmentation. Nature Neuroscience, 3, 622-627.

Yeshurun, Y., \& CARRasco, M. (2008). The effects of transient attention on spatial resolution and the size of the attentional cue. Perception \& Psychophysics, 70, 104-113.

Yeshurun, Y., Montagna, B., \& Carrasco, M. (2008). On the flexibility of sustained attention and its effects on a texture segmentation task. Vision Research, 48, 80-95.

(Manuscript received December 15, 2009; revision accepted for publication March 25, 2010.) 